Pacific Journal of Mathematics

ON VISUAL HULLS

G. Lamar and P. Mani 


\section{ON VISUAL HULLS}

\section{G. LARMAN AND P. MANI}

The concept of visual hull has been introduced by $\mathbf{G}$. $H$. Meisters and S. Ulam. In the following article we study a few of the problems arising from this notion and, in particular, establish (Theorem 3 ) a conjecture of W. A. Beyer and S. Ulam.

Let $C$ be a set in $R^{n}$ and $1 \leqq j \leqq n-1$. Then the $j^{\text {th }}$ visual hull $H_{j}(C)$ of $C$ is defined to be the largest set whose $j^{\text {th }}$ projections are contained in those of $C$. Alternatively, $H_{j}(C)$ is the set of points $x$ in $R^{n}$ such that each $(n-j)$-flat through $x$ contains a point of $C$. Let $G_{j}^{n}$ denote the Grassmannian of $j$-subspaces in $R^{n}$ with $\mu_{j}\left(G_{j}^{n}\right)=1$ for the usual measure $\mu_{j}$ associated with $G_{j}^{n}$ regarded as a metric $0_{n}$ factorspace. (For further information about $\mu_{j}$ compare, for example, [3]). The $j^{\text {th }}$ virtual hull $V_{j}(C)$ of $C$ is defined to be the set of points $x \in R^{n}$ such that almost all (with respect to $\left.\mu_{n-j}\right)(n-j)$-flats through $x$ contain a point of $C$. Thus, if $n=3, j=2, H_{2}(C)\left(V_{2}(C)\right)$ corresponds to those points in $R^{3}$ which are photographically indistinguishable (with probability one) from $C$. A $j^{\text {th }}$ minimal hull of $C$ in $R^{n}$ is a minimal set in $R^{n}$ whose $j^{\text {th }}$ projections coincide with those of $C$. In [2] the announced purpose of the paper was to disprove the conjecture that $H_{j}(C)-C$ is connected to $C$, i.e., $\nexists$ disjoint open sets $U, V$ such that $U \supset H_{j}(C)-C \neq \varnothing$ and $V \supset C \neq \varnothing$. To this we remark that a simple counterexample can be obtained by considering the closed set $C$ formed by removing the relative interiors of alternate sides of a regular hexagon inscribed in a plane circle with centre $a$. The first visual hull $H_{1}(C)$ is then $C \cup\{a\}$.

\section{Visual hulls of unions of polytopes.}

Theorem 1. Let $A_{1}, \cdots, A_{j+1}$ be spherically convex, closed subsets (not necessarily nonempty) of the sphere $S^{n-1}$, such that each ( $n-$ $j-1)$-subsphere of $S^{n-1}$ has a nonempty intersection with $\bigcup_{i=1}^{j+1} A_{i}$. Then $A_{1} \cap \cdots \cap A_{j+1} \neq \varnothing$. (so, that, in particular, each set $A_{i}$ is nonempty).

REMARK. $\quad S^{n-1}$ is the unit sphere of $R^{n}$ and an $(n-j-1)$-subsphere of $S^{n-1}$ is the intersection of an $n-j$ subspace with $S^{n-1}$. A set $C \subset S^{n-1}$ is spherically convex if $C$ is contained in an open hemisphere of $S^{n-1}$ and, if $x, y \in C$ then $C$ contains the minor arc on the 1-subsphere determined by $x, y$ and 0 (the centre of $S^{n-1}$ ).

Proof. The case $n=1$ is trivial. We assume inductively that 
the result is true for all $n^{\prime}<n$ and it remains to prove the result for $j+1$ sets on $S^{n-1}$. Assume on the contrary that there exist spherically convex closed subsets $A_{1}, \cdots, A_{j+1} \subset S^{n-1}$ such that

$$
T \cap\left(A_{1} \cup \cdots \cup A_{j+1}\right) \neq \varnothing
$$

for each $(n-j-1)$-subsphere $T$ of $S^{n-1}$, and $A_{1} \cap \cdots \cap A_{j+1}=\varnothing$. Let $A=A_{1} \cap \cdots \cap A_{j}$. Then $A, A_{j+1}$ are disjoint spherically convex closed subsets of $S^{n-1}$, and there exists an $(n-2)$-subsphere $S^{\prime}$ of $S^{n-1}$ which separates $A$ and $A_{j+1}$ and such that $S^{\prime} \cap A=\varnothing, S^{\prime} \cap A_{j+1}=\varnothing$. Set $A_{i}^{\prime}=A_{i} \cap S^{\prime}(1 \leqq i \leqq j)$. Then each $A_{i}^{\prime}$ is a spherically convex closed subset of $S^{\prime}$ and, since $A_{j+1} \cap S^{\prime}=\varnothing$, each $(n-j-1)$-subsphere of $S^{\prime}$ has a nonempty intersection with $A_{1}^{\prime} \cup \cdots \cup A_{j}^{\prime}$. Hence by the inductive assumption $A_{1}^{\prime} \cap \cdots \cap A_{j}^{\prime}=A \cap S^{\prime} \neq \varnothing$; contradiction.

TheOREM 2. In $R^{n}$ let $C_{1}, \cdots, C_{j+1}$ be $j+1$ compact convex sets. If $x \in H_{j}\left(\bigcup_{i=1}^{j+1} C_{i}\right)$ then either $x \in \bigcup_{i=1}^{j+1} C_{i}$ or there exists a halfine $l$ emanating from $x$ such that $l \cap C_{i} \neq \varnothing, 1 \leqq i \leqq j+1$.

CoROllaRY. In $R^{n}$ let $C_{1}, \cdots, C_{j+1}$ be compact convex sets. Then asufficient condition for $H_{j}\left(\bigcup_{i=1}^{j+1} C_{i}\right)=\bigcup_{i=1}^{i+1} C_{i}$ is that the sets do not have a common transversal.

Proof. On $S^{n-1}$ define $j+1$ spherically convex closed subsets $A_{1}, \cdots, A_{j+1}$ so that $u \in A_{i}$ if $u \in S^{n-1}$ and the half line $\{x+\lambda u \mid \lambda \geqq 0\}$ meets $C_{i}$. Then, as $x \in H_{j}\left(\bigcup_{i=1}^{j+1} C_{i}\right)$ each $(n-j-1)$-subsphere of $S^{n-1}$ has a nonempty intersection with $\bigcup_{i=1}^{j+1} A_{i}$. And so, by Theorem 1, there exists $u \in \bigcap_{i=1}^{j+1} A_{i}$, i.e., the halfline $\{x+\lambda u \mid \lambda \geqq 0\}$ meets each of $C_{1}, \cdots, C_{j+1}$.

THeOREM 3. In $R^{n}$ let $C_{1}, \cdots, C_{j+1}$ be nonempty compact convex sets. Then the number of components of $H_{j}\left(\bigcup_{i=1}^{j+1} C_{i}\right)$ is at most $j+1$ with equality if and only if $C_{1}, \cdots, C_{j+1}$ are pairwise disjoint.

Proof. By Theorem 2, if $x \in H_{j}\left(\bigcup_{i=1}^{j+1} C_{i}\right)-\bigcup_{i=1}^{j+1} C_{i}$, then there exists a halfline $l=\{x+\lambda u \mid \lambda \geqq 0\}$ such that $l$ meets each of

$$
C_{1}, \cdots, C_{j+1} \text {. }
$$

Then $x+\alpha_{k} u \in C_{k}$ for some $\alpha_{k}>0$. We set $\alpha=\min \left\{\alpha_{k} \mid 1 \leqq k \leqq j+1\right\}$ and want to show that $x+\lambda u \in H_{j}\left(\bigcup_{i=1}^{j+1} C_{i}\right)$ for all $\lambda$ with $0 \leqq \lambda \leqq \alpha$. Set $y=x+\lambda u$ and let $P$ be an $(n-j)$-subspace. As $x \in H_{j}\left(\bigcup_{i=1}^{j+1} C_{i}\right)$ there exists $i$ such that the $(n-j)$-flat $x+P$ meets $C_{i}$ at $v$, say. Set $z=x+\alpha_{i} u \in C_{i}$. Then, as $y$ lies between $x$ and $z$ on $l$, there exists $\mu, 0 \leqq \mu \leqq 1$, such that $y=\mu x+(1-\mu) z$. Then the $(n-j)$ flat $y+P$ through $y$ contains the point $\mu v+(1-\mu) z$ of $C_{i}$. As $P$ 
was arbitrary we conclude that $y \in H_{j}\left(\bigcup_{i=1}^{j+1} C_{i}\right)$ and hence that $x+$ $\lambda u \in H_{j}\left(\bigcup_{i=1}^{j+1} C_{i}\right)$ for $0 \leqq \lambda \leqq \alpha$. Hence, if $x \in H_{j}\left(\bigcup_{i=1}^{j+1} C_{i}\right)$ then $x$ is connected, via a line segment in $H_{j}\left(\bigcup_{i=1}^{j+1} C_{i}\right)$, to at least one of the sets $C_{i}$. Hence $H_{j}\left(\bigcup_{i=1}^{j+1} C_{i}\right)$ has at most $j+1$ components with equality only if the $C_{i}$ 's are disjoint. If the sets $C_{1}, \cdots, C_{j+1}$ are pairwise disjoint then in order to show that $H_{j}\left(\bigcup_{i=1}^{j+1} C_{i}\right)$ has exactly $j+1$ components it is enough to show that for each $k, 1 \leqq k \leqq j+1$, there exist disjoint open sets $U_{k}, V_{k}$ such that $U_{k} \cup V_{k} \supset H_{j}\left(\bigcup_{i=1}^{j+1} C_{i}\right)$ and $U_{k} \supset C_{k}, V_{k} \supset\left\{C_{1} \cup \cdots \cup C_{k-1} \cup C_{k+1} \cup \cdots \cup C_{j+1}\right\}$. We suppose, without loss of generality, that $k=1$. For $i=2, \cdots, j+1$ let $H_{i}$ denote a hyperplane which strictly separates $C_{1}$ from $C_{i}$, and let $H_{i}^{0}$ be the open halfspace bounded by $H_{i}$ and containing $C_{1}$. We can assume that the $H_{i}$ 's are in general position. Set $U_{1}=\bigcap_{i=2}^{j+1} H_{i}^{0}, V_{1}=R^{n}-\bar{U}_{1}$. Then $U_{1}$ and $V_{1}$ are disjoint open sets, $C_{1} \subset U_{1}, \bigcup_{i=2}^{j+1} C_{i} \subset V_{1}$. It remains to show that $H_{j}\left(\bigcup_{i=1}^{j+1} C_{i}\right) \subset U_{1} \cup V_{1}$, and it is enough to show that $\left(\bar{U}_{1} \cap \bar{V}_{1}\right) \cap H_{j}\left(\bigcup_{i=1}^{j+1} C_{i}\right)=\varnothing$. Since the $H_{i}$ 's are in general position, their intersection $\bigcap_{i=2}^{j+1} H_{i}$ is an $(n-j)$-dimensional flat $L$. Let $I$ be the $j$-dimensional subspace orthogonal to $L$. If $M$ is any subset of $R^{n}$ we denote by $\operatorname{proj}_{I} M$ the set of all points $x \in I$ for which the flat $L_{x}$, which is parallel to $L$ and contains $x$, has a nonempty intersection with $M . \operatorname{proj}_{I} U_{1}$ and $\operatorname{proj}_{I} V_{1}$ are two open sets in $I$ with common boundary $\operatorname{proj}_{I}\left(\bar{U}_{1} \cap \bar{V}_{1}\right)$. As $\operatorname{proj}_{I} C_{1} \subset \operatorname{proj}_{I} U_{1}, \operatorname{proj}_{I} \bigcup_{i=2}^{j+1} C_{i} \subset \operatorname{proj}_{I} V_{1}$ it follows that $\left(\operatorname{proj}_{I}\left(\bar{U}_{1} \cap \bar{V}_{1}\right)\right) \cap\left(\operatorname{proj}_{I} \bigcup_{i=1}^{j+1} C_{i}\right)=\varnothing$. Now, if $z$ is an arbitrary point in $\bar{U}_{1} \cap \bar{V}_{1}$ it follows that $L_{z} \cap\left(\bigcup_{i=1}^{j+1} C_{i}\right)=\varnothing$, and since $\operatorname{dim} L_{z}=n-j$, we find, by the definition of $H_{j}$, that $z$ does not belong to $H_{j}\left(\bigcup_{i=1}^{j+1} C_{i}\right)$. Therefore $\left(\bar{U}_{1} \cap \bar{V}_{1}\right) \cap H_{j}\left(\bigcup_{i=1}^{j+1} C_{i}\right)=\varnothing$.

REMARKs. The proof of Theorem 3 also shows that any component of $H_{j}\left(\bigcup_{i=1}^{j+1} C_{i}\right)$ has the property that any two points of it can be joined by a broken line in it, consisting of at most 3 segments. Hence it is natural to ask: When are these components convex? (supposing now that the $C_{i}$ 's are disjoint). In [1] W. A. Beyer has shown an example of three (nondisjoint) polytopes $C_{i}$ in $R^{3}$ such that $H_{2}\left(C_{1} \cup C_{2} \cup C_{3}\right)$ is not a polyhedron. We don't know whether a similar construction would be possible with disjoint polytopes. Let us mention here a few more technical terms. If $M$ is any subset of $R^{n}$, we denote by aff $M$ the affine hull of $M$ and by conv $M$ the convex hull of $M$. relint $M$ means the interior of $M$ with respect to the natural topology in aff $M$. By the dimension $\operatorname{dim} M$ of $M$ we understand the algebraic dimension of the flat aff $M$. A polytope is the convex hull of some finite set. If $P \subset E^{n}$ is a convex set we denote by ext $P$ the set of extreme points of $P$ and by $\exp P$ the set of its exposed points. For an exact definition of these terms the reader may compare, for example, the introductory chapters of [4]. 
Theorem 4. (i) In $R^{n}$ let $C_{1}, C_{2}$ be compact convex sets. Then $H_{1}\left(C_{1} \cup C_{2}\right)$ is the union of at most two convex components which are polytopes whenever $C_{1}$ and $C_{2}$ are polytopes.

(ii) There exist in $R^{3}$ three disjoint polytopes such that one of the components of the second visual hull of their union is not convex.

Lemma 1. Let $C_{1}, C_{2}$ be n-dimensional polytopes in $R^{n}$. If $a \notin H_{1}\left(C_{1} \cup C_{2}\right)$ there exists a hyperplane $H$ such that

(1) $a \notin H, H$ separates a from $C_{1}$

(2) $H \cap C_{i}=\varnothing$ or $H$ supports $C_{i}(i=1,2)$

(3) aff $\left(H \cap\left(C_{1} \cup C_{2}\right)\right)=H$.

Proof of Lemma 1. The case $n=1$ is trivial, and we assume $n \geqq 2$. If there exists a hyperplane $P$ through $a$ which does not meet $C_{1} \cup C_{2}$ and does not separate $C_{1}$ and $C_{2}$ then conv $\left(C_{1} \cup C_{2}\right)$ is an $n$-dimensional polytope not containing $a$, and the lemma follows from standard results on polytopes. Hence it can be supposed that there is a hyperplane $H$ for which (1) and also $\left(2^{\prime}\right)$ : $H$ separates $C_{1}$ and $C_{2}$ holds. We choose $H$ in the set $\mathfrak{S}$ of hyperplanes for which (1) and (2') holds. We assume that $h=\operatorname{dim}$ aff $T$ is maximal, where $T=H \cap\left(C_{1} \cup C_{2}\right)$. Obviously $h \geqq 0$. If $h<n-1$, let $F \subset H$ be an $(n-2)$-dimensional hyperplane in $H$ containing $T$, and denote by $\pi$ : $R^{n} \rightarrow E$ the projection along $F$ onto a 2-dimensional flat $E$ orthogonal to $F$. It is easy to see that there is a line $L$ in $E$ such that: $(\alpha)$ : the singleton $\pi(T)$ is contained in $L . \quad(\beta): \pi(a) \notin L, L$ separates $\pi(a)$ from the polygon $\pi\left(C_{1}\right)(\gamma): L$ separates $\pi\left(C_{1}\right)$ and $\pi\left(C_{2}\right)$.

$(\delta)$ aff $\left(L \cap\left(\pi\left(C_{1}\right) \cup \pi\left(C_{2}\right)\right)=L\right.$.

(Notice that the conditions $(\alpha)-(\gamma)$ are fulfilled by $\pi(H))$. The hyperplane $\pi^{-1}(L)$ of $E^{n}$ intersects $C_{1} \cup C_{2}$ in a set $S$ with dimaff $S=$ $h+1$. Since $S \in \mathfrak{S}$ this contradicts the maximality of $h$. Hence the lemma is established.

Proof of Theorem 4. (i) We first prove the result when $C_{1}, C_{2}$ are $n$-dimensional polytopes. If $C_{1} \cap C_{2} \neq \varnothing$ then

$$
H_{1}\left(C_{1} \cup C_{2}\right)=\operatorname{conv}\left(C_{1} \cup C_{2}\right) \text {, }
$$

which is a polytope. We suppose therefore that $C_{1} \cap C_{2}=\varnothing$. Let $\left\{H_{i}\right\}_{i=1}^{m}$ be the finite set of those hyperplanes which do not contain an interior of $C_{j}(j=1,2)$ and for which $\operatorname{dim}\left(H_{i} \cap\left(C_{1} \cup C_{2}\right)\right)=n-1$. By $C_{j}^{*}$ we denote the (finite) intersection of those closed half spaces which contain $C_{j}$ and whose bounding hyperplane is amongst $\left\{H_{i}\right\}_{i=1}^{m}, j=1,2$. Then $C_{\jmath}^{*}$ is polyhedral and, since $C_{1}, C_{2}$ are compact, $C_{\jmath}^{*}$ is a polytope, 
$j=1$, 2. We show that $H_{1}\left(C_{1} \cup C_{2}\right)=C_{1}^{*} \cup C_{2}^{*}$. Suppose that $x^{*} \notin C_{1}^{*} \cup C_{2}^{*}$. Then there exist closed halfspaces $H_{1}^{*}, H_{2}^{*}$ with bounding hyperplanes $H_{1}, H_{2}$ amongst $\left\{H_{i}\right\}_{i=1}^{m}$ such that $x^{*} \notin H_{1}^{*} \supset C_{1}, x^{*} \notin H_{2}^{*} \supset C_{2}$. If

$$
x^{*} \in H_{1}\left(C_{1} \cup C_{2}\right), H_{1} \quad \text { and } \quad H_{2}
$$

must separate $C_{1}$ and $C_{2}$. Consider $H_{1}$ and the two disjoint compact sets $H_{1} \cap C_{1}, H_{1} \cap C_{2}$ in $H_{1}$. There exists an $n-2$ dimensional flat $L$ in $H_{1}$ which strictly separates $H_{1} \cap C_{1}$ and $H_{1} \cap C_{2}$. By slightly rotating $H_{1}$ about $L$ in the appropriate direction we obtain a hyperplane $H_{1}^{\prime}$ which strictly separates $C_{1}$ and $C_{2}$ as well as $x^{*}$ and $C_{1}$. Similarly we can obtain a hyperplane $H_{2}^{\prime}$ which strictly separates $C_{1}$ and $C_{2}$, and $x^{*}$ and $C_{2}$. We may suppose that $H_{1}^{\prime}, H_{2}^{\prime}$ are not parallel and so $H_{1}^{\prime} \cap H_{2}^{\prime}$ is an $n-2$ flat. Suppose, without loss of generality, that $H_{1}^{\prime}=\{x|\langle x, \xi\rangle=\alpha\rangle 0\}, H_{2}=\{x|\langle x, \eta\rangle=\beta\rangle 0\}$. Then

$$
\begin{aligned}
& C_{1} \subset\{x \mid\langle x, \xi\rangle>\alpha\} \cap\{x \mid\langle x, \eta\rangle>\beta\} \\
& C_{2} \subset\{x \mid\langle x, \eta\rangle\langle\alpha\} \cap\{x \mid\langle x, \eta\rangle\langle\beta\} .
\end{aligned}
$$

Consider the hyperplane $H:\{x \mid\langle x, \lambda \xi+(1-\lambda) \eta\rangle=0\}$, where $\lambda \alpha+$ $(1-\lambda) \beta=0$ and $0<\lambda<1$. Then $x^{*} \in H$ and, using the above inequalities, $C_{i} \cap H=\varnothing, i=1,2$. Hence $x^{*}$ is not in $H_{1}\left(C_{1} \cup C_{2}\right)$, and we have $H_{1}\left(C_{1} \cup C_{2}\right) \subset C_{1}^{*} \cup C_{2}^{*}$. Conversely, if $x^{*} \in C_{1}^{*} \cup C_{2}^{*}-H_{1}\left(C_{1} \cup C_{2}\right)$, suppose without loss of generality that $x^{*} \in C_{1}^{*}$. Then, by Lemma 1 , there exists a hyperplane $H$ amongst $\left\{H_{i}\right\}_{i=1}^{m}$ which does not contain $x^{*}$ and which separates $x^{*}$ from $C_{1}$. Then, if $H^{*}$ donotes the closed halfspace containing $C_{1}$ whose bounding hyperplane is $H, x^{*} \notin H^{*}$ and so $x^{*} \in C_{1}^{*}$; contradiction. And so $H_{1}\left(C_{1} \cup C_{2}\right)=C_{1}^{*} \cup C_{2}^{*}$, which is the union of two polytopes. If $C_{1}, C_{2}$ are compact convex sets we choose decreasing sequences $\left\{P_{1}^{n}\right\}_{n=1}^{\infty},\left\{P_{2}^{n}\right\}_{n=1}^{\infty}$ of polytopes such that $C_{i}=\bigcap_{n=1}^{\infty} P_{i}^{n}$, $i=1,2$. Then, using the above notation,

$$
H_{1}\left(C_{1} \cup C_{2}\right)=\bigcap_{n=1}^{\infty} P_{1}^{n *} \cap \bigcap_{n=1}^{\infty} P_{2}^{n *} .
$$

(ii) Let $W$ be the cube $\left\{x=\left(x_{1}, x_{2}, x_{3}\right) \mid-1 \leqq x_{i} \leqq 1, i=1,2,3\right\}$ in $R^{3}$, and denote by $W_{i}$ the facet of $W$ defined by $x_{i}=1$. Set $C_{1}=W_{1}$, $C_{2}=2 W_{2}, C_{3}=3 W_{3}$. Let $B_{i}(1 \leqq i \leqq 3)$ be the components of $H_{2}\left(\bigcup_{i=1}^{3} C_{i}\right)$, where the indices are chosen such that, for all $i, C_{i} \subset B_{i}$. Clearly $(0,0,0) \in B_{1}$ as does, of course, the point $(1,-1,-1) \in B_{1} \cap C_{1}$. However we show that the line segment $m:\{x=\lambda(1,-1,-1) \mid 0<\lambda<1\}$ is not in $B_{1}$. Now $C_{1} \cup C_{2}$ is contained in the halfspace $\{x \mid\langle x,(0,1,1)\rangle \geqq 0\}$ whose bounding hyperplane $P$ passes through the points $(0,0,0)$, $(1,-1,1)$ and $(-1,-1,1) ; P \cap$ aff $W_{1}$ is a line in direction $(0,-1,1)$. If $y \in m$, then $y=\mu(1,-1,-1)$ for some $\mu, 0<\mu<1$. Consider the line $l=y+\{\lambda(0,-1,1) \mid \lambda$ real $\}$. If $z=\left(z_{1}, z_{2}, z_{3}\right) \in l$ then $z_{1}=\mu<1$, 
i.e., $z \notin C_{1}$. Also $\langle z,(0,1,1)\rangle=-2 \mu<0$ which means that $z \notin C_{1} \cup C_{2}$. Therefore $l$ does not meet $C_{1} \cup C_{2} \cup C_{3}, m$ does not belong to $B_{1}$, and $B_{1}$ is not convex.

In [6] V. L. Klee proved that if all $j^{\text {th }}$ projections of a compact convex body $C$ in $R^{n}$ ( $j$ fixed $\geqq 2$ ) are polytopes, then $C$ is a polytope. As a partial analogue to this for unions of two convex bodies we prove

TheOREm 5. Let $C_{1}, C_{2}$ be two disjoint compact convex bodies in $R^{n}$ such that each $j^{\text {th }}$ projection of $C_{1} \cup C_{2}(j$ fixed $\geqq 2)$ is the union of two polytopes. Then (i) $\operatorname{ext}\left(C_{i}\right)=\exp \left(C_{i}\right)$ and $\operatorname{ext}\left(C_{i}\right)$ is countable $(i=1,2)$ but (ii) ext $\left(C_{i}\right)$ is not necessarily finite.

Proof. Let $a$ be an extreme point of $C_{1}$ and we suppose, without loss of generality, that $a=0$, the origin of $R^{n}$. Then, to prove (i) it is enough to prove that the convex cone $K$ of outward normals to $C_{1}$ at 0 is $n$-dimensional. We assume that $\operatorname{dim} K \leqq n-1$ so that $K$ is contained in an $(n-1)$-subspace $P_{1}$, and seek a contradiction. Let $P_{2}$ be an $(n-1)$-subspace which supports $C_{1}$ at 0 . Of course $P_{1} \neq P_{2}$. We can choose an $(n-1)$-subspace $P_{3}$ so that there exists a translate of $P_{3}$ which strictly separates $C_{1}$ and $C_{2}$ and such that the normal to $P_{3}$ at 0 intersects $P_{1}$ only at 0 . Then $P_{2} \cap P_{3}$ is a subspace of dimension at least $n-2$ and we choose an $n-j$ subspace $Q$ in $P_{2} \cap P_{3}$. The orthogonal complement $S$ of $Q$ in $R^{n}$ is a $j$-dimensional subspace which meets $P_{1}$ in a $(j-1)$-subspace. The projection of $C_{1} \cup C_{2}$ onto $S$ is the union of two polytopes. Further, as $P_{3} \cap C_{2}=\varnothing, 0$ is at positive distance from proj $C_{2}$. As 0 is an extreme point of proj $C_{1}$, it follows that 0 is a locally polyhedral extreme point for $\operatorname{proj} C_{1}$. Hence, in $S$, the cone of outward normals to proj $C_{1}$ at 0 is $j$-dimensional. Further, any $(j-1)$-plane $H$ of support in $S$ to proj $C_{1}$ at 0 can be extended to an $(n-1)$-plane of support $H+Q$ in $R^{n}$ to $C_{1}$ at 0 . Also, the outward normals to these planes form a $j$-dimensional convex cone lying in $S$. Hence $j=\operatorname{dim}(K \cap S)=\operatorname{dim}\left(P_{1} \cap S\right)=j-1$; contradiction. And so (i) is proved.

To prove (ii) we construct an example in $R^{3}$ of two convex bodies $C_{1}, C_{2}$, both of which have a countable infinity of extreme points but, nevertheless, each 2-projection of $C_{1} \cup C_{2}$ is the union of two convex polygons. Let $l=\left\{x \mid x_{1}=x_{2}=0,-1 \leqq x_{2} \leqq 1\right\}$ be a line segment and $S=\left\{x \mid\left(x_{1}-1\right)^{2}+x_{2}^{2}=1, x_{3}=0\right\}$ a plane circle. By $T$ we denote the set of those points on $S$ with $x_{2}$-coordinate ${ }^{ \pm}(1 / n)$ for $n=1,2, \cdots$. We take $C_{1}=\operatorname{conv}\{l \cup T\}$, which is a compact convex body in $R^{3}$ with extreme points $T \cup\{(0,0,-1),(0,0,1)\}$. It is easily seen that there is precisely one 2-projection of $C_{1}$ which is not a convex polygon, and that is in the direction $(0,0,1)$. Further the only limit point of extreme points of this projection is $(0,0,0)$. Define $C_{2}$ as a disjoint copy of 
$C_{1}$ formed by placing $C_{2}$ above $C_{1}$ in such a way that their respective major lines pierce the centres of their respective circles. From above, every 2-projection of $C_{1} \cup C_{2}$ is the union of two convex polygons and and both $C_{1}$ and $C_{2}$ are compact bodies with a countable infinity of extreme points.

3. Visual hulls of more general sets. The following problem can be formulated.

Is the visual (virtual) (minimal) hull of a borel (analytic) set in $R^{n}$ necessarily borel (analytic)?

The answer is affirmative (Theorem 6) for virtual hulls and negative (Theorem 7) for minimal hulls. Whilst it is not true (Theorem 8) that the $j^{\text {th }}$ visual hull of a borel set is necessarily borel, we have been unable to decide whether or not the $j^{\text {th }}$ visual hull of a borel or of an analytic set is always analytic, except in the cases covered by Theorem 9. It is possible also that the $j^{\text {th }}$ visual hull of a convex borel (analytic) set is a borel (analytic) set, and we include some partial results (Theorem 9) in this direction. As before we denote by $G_{j}^{n}$ the Grassmannian of $j$-subspaces of $R^{n}$ and by $\mu_{j}$ the invariant (with respect to $0_{n}$ acting in the usual way on $\left.G_{j}^{n}\right)$ measure normalised so that $\mu_{j}\left(G_{j}^{n}\right)=1$.

Lemma 2. Let $A$ be an analytic set in $R^{n}$ and denote by $A^{*}$ the set of those $j$-subspaces in $G_{j}^{n}$ which meet $A$. Then

(i ) $A^{*}$ is an analytic set in $G_{j}^{n}$ and hence $A^{*}$ is $\mu_{j}$ measurable.

(ii) If $\mu_{j}\left(A^{*}\right)>a$ then there exists a compact subset $A^{\prime}$ of $A$ such that $\mu_{j}\left(A^{* *}\right)>a$.

(iii) If $A_{1} \subset A_{2} \subset \cdots$ is an increasing sequence of analytic sets in $R^{n}$ then $\mu_{j}\left(\bigcup_{i=1}^{\infty} A_{i}\right)^{*}=\lim _{i \rightarrow \infty} \mu_{j}\left(A_{i}^{*}\right)$.

(iv) If $A_{1} \supset A_{2} \supset \cdots$ is a decreasing sequence of analytic sets in $R^{n}$ then $\mu_{j}\left(\bigcap_{i=1}^{\infty} A_{i}\right)^{*}=\lim _{i \rightarrow \infty} \mu_{j}\left(A_{i}^{*}\right)$.

Proof. (i ) Let $I$ be the set of irrational numbers in $[0,1]$ and, if $i=\left(i_{1}, \cdots, i_{n}, \cdots\right)$ is a typical member of $I$ expressed as a continued fraction, set $i \mid n=\left(i_{1}, \cdots, i_{n}\right)$. Then, as $A$ is analytic, it can be represented as $A=\sum_{i \in I} \bigcap_{n=1}^{\infty} A(i \mid n)$ where the sets $A(i \mid n)$ form, for each fixed $i$, a decreasing sequence of compact subsets of $R^{n}$. Then $A^{*}=\sum_{i \in I} \bigcap_{n=1}^{\infty} A^{*}(i \mid n)$. As each $A^{*}(i \mid n)$ is a compact subset of $G_{3}^{n}$, we conclude that $A^{*}$ is an analytic set.

(ii) If $\mu_{j}\left(A^{*}\right)>a+\delta$ with $\delta>0$, then we can choose $m_{1}, 1 \leqq$ $m_{1}<\infty$, such that if $I_{1}$ denotes the set of irrational numbers

$$
i=\left(i_{1} \cdots i_{n} \cdots\right)
$$

with $1 \leqq i_{1} \leqq m_{1}$ and $A_{1}^{*}=\sum_{i \in I_{1}} \bigcap_{n=1}^{\infty} A^{*}(i \mid n)$ then $\mu_{j}\left(A_{1}^{*}\right)>a+\delta$. 
Proceeding by induction we may define natural numbers $m_{p}, 1 \leqq p<\infty$, such that if $I_{q}$ denotes the subset of those irrationals $i$ with $1 \leqq i_{p} \leqq m_{p}$ for $p=1, \cdots q$, and $A_{q}^{*}=\sum_{i \in I_{q}} \bigcap_{n=1}^{\infty} A^{*}(i \mid n)$ then $\mu_{j}\left(A_{q}^{*}\right)>a+\delta$. Let $I^{\prime}$ be the compact subset of $[0,1]$ defined as the set of those irrational numbers $i$ for which $1 \leqq i_{p} \leqq m_{p}$ for $p=1,2, \cdots$, and

$$
A^{* *}=\sum_{i \in L^{\prime}} \bigcap_{n=1}^{\infty} A^{*}(i \mid n) \text {. }
$$

Then $\bigcap_{q=1}^{\infty} A_{q}^{*}=A^{*}$ and so $\mu_{j}\left(A^{\prime *}\right) \geqq a+\delta>a$. Also

$$
A^{\prime}=\sum_{i \in 1^{\prime}} \bigcap_{n=1}^{\infty} A(i \mid n)
$$

is a compact subset of $A$, as $I^{\prime}$ is a compact subset of $I$.

(iii) $\mu_{j}\left(\bigcup_{i=1}^{\infty} A_{i}\right)^{*}=\mu_{j}\left(\bigcup_{i=1}^{\infty} A_{i}^{*}\right)=\lim _{i-\infty} \mu_{j}\left(A_{i}^{*}\right)$.

(iv) Clearly $\mu_{j}\left(\bigcap_{i=1}^{\infty} A_{i}\right)^{*} \leqq \lim _{i-\infty} \mu_{j}\left(A_{i}^{*}\right)$. Now set $\mu_{j}\left(\bigcap_{i=1}^{\infty} A_{i}\right)^{*}=a$. and suppose $\lim _{i \rightarrow \infty} \mu_{j}\left(A_{i}^{*}\right)>a+\varepsilon$, for some positive number $\varepsilon$. By (ii) we find a compact set $B_{1} \subset A_{1}$ such that $\mu_{j}\left(B_{1}^{*}\right) \geqq \mu_{j}\left(A_{1}^{*}\right)-\varepsilon / 2$. Now we have $A_{2}^{*}=\left(B_{1} \cap A_{2}\right)^{*} \cup\left(A_{2}^{*}-B_{1}^{*}\right)$, where

$$
A_{2}^{*}-B_{1}^{*}=\left\{F \in G_{j}^{n} \mid F \cap A_{2} \neq \varnothing \text {, but } F \cap B_{1}=\varnothing\right\} \text {. }
$$

Since $A_{2}^{*} \subset A_{1}^{*}$ we derive further $A_{2}^{*} \subset\left(B_{1} \cap A_{2}\right)^{*} \cup\left(A_{1}^{*}-B_{1}^{*}\right)$, or $\mu_{j}\left(A_{2}^{*}\right) \leqq \mu_{j}\left(B_{1} \cap A_{2}\right)^{*}+\varepsilon / 2$. Since $B_{1} \cap A_{2}$ is analytic there exists, again by (ii), a compact set $B_{2} \subset\left(B_{1} \cap A_{2}\right)$ such that

$$
\mu_{j}\left(B_{2}\right)^{*} \geqq \mu_{j}\left(B_{1} \cap A_{2}\right)^{*}-\varepsilon / 4
$$

and consequently $\mu_{j}\left(B_{2}\right)^{*} \geqq \mu_{j}\left(A_{2}\right)^{*}-(\varepsilon / 2+\varepsilon / 4)$. Continuing this process we obtain a decreasing sequence $\left\{B_{i}\right\}_{i=1}^{\infty}$ of compact subsets of $R^{n}$ such that $B_{i} \subset A_{i}, i=1,2, \cdots$, and $\mu_{j}\left(B_{i}^{*}\right) \geqq \mu_{j}\left(A_{i}^{*}\right)-\sum_{p=1}^{i} \varepsilon /\left(2^{p}\right)$. Then $\bigcap_{i=1}^{\infty} B_{i}^{*}=\left(\bigcap_{i=1}^{\infty} B_{i}\right)^{*} \subset\left(\bigcap_{i=1}^{\infty} A_{i}\right)^{*}$, and $\mu_{j}\left(\bigcap_{i=1}^{\infty} B_{\imath}^{*}\right)=\lim _{i \rightarrow \infty} \mu_{j}\left(B_{i}^{*}\right) \leqq a$; but also $\lim _{i-\infty} \mu_{j}\left(B_{i}^{*}\right) \geqq \lim _{i \rightarrow \infty} \mu_{j}\left(A_{i}^{*}\right)-\varepsilon$. Combining the last two inequalities we find $\lim _{i \rightarrow \infty} \mu_{\jmath}\left(A_{i}\right) \leqq a+\varepsilon$, a contradiction.

Theorem 6. Let $C$ be a borel (analytic) set in $R^{n}$. Then the $j^{\text {th }}$ virtual hull $V_{j}(C)$ is a borel (analytic) set.

Proof. Suppose first that $C$ is a borel set in $R^{n}$, and we need to show that $V_{j}(C)$ is a borel set. If $D$ is a subset of $R^{n}$ and $x \in R^{n}$, let $D[x, n-j]$ denote the set of those $n-j$ subspaces $F$ in $G_{n-j}^{n}$ such that $(x+F) \cap D \neq \varnothing$. If $0<\lambda<1$ let $D(n-j, \lambda)$ be the set of all $x$ in $R^{n}$ such that $\mu_{n-j}(D[x, n-j])>\lambda$. Let $B$ denote the largest family of subsets of $R^{n}$ such that $D \in B$ if (i) $D$ is a borel set in $R^{n}$. (ii) $D(n-j, \lambda)$ is a borel set for all $\lambda, 0<\lambda<1$. We shall prove that $B$ coincides with the family of borel subsets of $R^{n}$, and it is enough 
to show that $B$ contains the open sets and is closed under the operations of increasing union and decreasing intersection. If $D$ is an open subset of $R^{n}$, then it is easy to see that $D(n-j, \lambda)$ is open for all $\lambda, 0<\lambda<1$, and so $B$ contains all the open sets. Now suppose that $\left\{E_{i}\right\}_{i=1}^{\infty}$ is an increasing sequence of sets in $B$ and set $E=\bigcup_{i=1}^{\infty} E_{i}$. We want to show that for each $\lambda, 0<\lambda<1$, the equality $E(n-j, \lambda)=\bigcup_{i=1}^{\infty} E_{i}(n-j, \lambda)$ holds. In order to do this we observe the following equivalences: $x \in E(n-j, \lambda) \leftrightarrow \mu_{n-j}(E[x, n-j])>\lambda \leftrightarrow \lim _{i \rightarrow \infty} \lambda_{n-j}\left(E_{i}[x, n-j]\right)>\lambda \leftrightarrow$ $x \in \bigcup_{i=1}^{\infty} E_{i}(n-j, \lambda)$. Here the first equivalence holds by definition, the second one follows directly from Lemma 2, (iii), if we observe that this lemma remains true if $M^{*}$ denotes, for each $M \subset R^{n}$, the set $M[x, n-j]$ ( $x \in R^{n}$ fixed). (The lemma itself is stated for the special case where $x$ is the origin of $R^{n}$.) The last equivalence again follows immediately from the definitions, we only have to observe that the sequence $\left\{E_{i}\right\}_{i=1}^{\infty}$ is increasing. Now suppose that $\left\{H_{i}\right\}_{i=1}^{\infty}$ is a decreasing sequence of subsets of $B$ and set $H=\bigcap_{i=1}^{\infty} H_{i}$. Suppose $\lambda$ fixed, $0<\lambda<1$, and let $m$ be a natural number such that $\lambda+1 / m<1$. Then, using (iv) of Lemma 2, we find by an argument analogous to the one above, $H(n-j, \lambda)=\bigcup_{p=m}^{\infty} \bigcap_{i=1}^{\infty} H_{i}(n-j, \lambda+1 / p)$. Hence $H(n-j, \lambda)$ is a borel set, and $H \in B$. Therefore, $B$ is the family of borel subsets of $R^{n}$ and so, in particular, $C \in B$. Further $V_{j}(C)=$ $\bigcap_{p=2}^{\infty} C(n-j, 1-(1 / p))$ and so $V_{j}(C)$ is a borel set.

To show that $V_{j}(A)$ is analytic whenever $A$ is analytic, we use the well known result that there exists an $F_{\sigma o}$ set $K$ in $R^{n+1}$ such that $A$ is the orthogonal projection proj $K$ of $K$ into $R^{n}$ (see, for example, [8]). Call an $(n-j+1)$-subspace $H$ of $R^{n+1}$ upright if $H$ has the form $\{\hat{H}+\lambda(0, \cdots, 0,1) \mid-\infty<\lambda<\infty\}$ where $\hat{H} \in G_{n-j}^{n}$. Let $U_{j+1}$ be the set of upright $(n-j+1)$-subspaces in $R^{n+1}$ with the measure $\mu^{\prime}$ induced by $\mu_{n-j}$ in the obvious manner. We can define $U_{j+1}(C)$ of a set $C$ in $R^{n+1}$ as the set of all those points $x$ in $R^{n+1}$ such that almost all (with respect to $\left.\mu^{\prime}\right)$ upright $(n-j+1$ )-flats through $x$ meet $C$. As above, it can been shown that $U_{j+1}(C)$ is a borel set whenever $C$ is a borel set. Clearly proj $U_{j+1}(K)=V_{j}(A)$ and, since the projection of a borel set is analytic, we conclude that $V_{j}(A)$ is an analytic subset of $R^{n}$.

Theorem 7. Let $C$ be an open convex subset of $R^{n}$. Then assuming the continuum hypothesis, $C$ contains a minimal $j^{\text {th }}$ hull $D$ such that every analytic subset of $D$ is countable. ${ }^{1}$

Proof. We assume the continuum hypothesis and let $\Omega$ be the

1 As the referee pointed out, Theorem 7 may be a special case of a much more general theorem on effective constructions. 
first uncountable ordinal. Let $\left\{A_{\xi}\right\}_{\xi<\Omega}$ be an enumeration of the analytic subsets of $R^{n}$ of $(n-j)$-dimensional measure zero; let $\left\{H_{\xi}\right\}_{\xi<\Omega}$ be an enumeration of the $(n-j)$-flats which meet $C$. Let $F$ be a fixed $(n-j)$-subspace of $R^{n}$ and denote by $\alpha$ a fixed set, which is not a point of $R^{n}$. We now choose a set $E=\left\{M_{\xi}\right\}_{\xi<\Omega}$ and a collection of translates $\left\{F_{\xi}\right\}_{\xi<\Omega}$ of $F$ inductively as follows. Take $M_{1} \in\left(H_{1}-A_{1}\right) \cap C$ and let $F_{1}$ be a translate of $F$ through $M_{1}$. Suppose now that $M_{\xi^{\prime}}, F_{\xi^{\prime}}$ have been defined for all $\xi^{\prime}<\xi$, where $\xi$ is some ordinal proceeding $\Omega$. If $H_{\xi}$ is a translate of $F$ we take $F_{\xi}=H_{\hat{\xi}}$ and consider two possibilities:

(a) If $\exists \xi^{\prime}<\xi$ such that $M_{\xi^{\prime}} \in H_{\xi}$ then we take $M_{\hat{\xi}}=\alpha$.

(b) If $\exists \xi^{\prime}<\xi$ such that $M_{\xi^{\prime}} \in H_{\xi}$ we choose $M_{\xi}$ in the set $\left(H_{\xi}-\right.$ $\left.\left(\bigcup_{\xi^{\prime}<\xi} H_{\xi^{\prime}} \cup \bigcup_{\xi^{\prime}<\xi} A_{\xi^{\prime}}\right)\right) \cap C$. Such a choice is possible as $H_{\xi} \cap C$ has positive $(n-j)$-dimensional measure whereas $H_{\xi} \cap\left(\bigcup_{\xi^{\prime}<\xi} H_{\xi^{\prime}} \cup \bigcup_{\xi^{\prime}<\xi} A_{\xi^{\prime}}\right)$ has zero $(n-j)$-dimensional measure, being a countable union of sets of measure zero. If $H_{\xi}$ is not a translate of $F$ we find, by similar arguments, that the set $\left(H_{\xi}-\left(\bigcup_{\xi^{\prime}<\xi} H_{\xi^{\prime}} \cup \bigcup_{\xi^{\prime}<\xi} A_{\xi^{\prime}} \cup \bigcup_{\xi^{\prime}<\xi} F_{\xi^{\prime}}\right)\right) \cap C$ is not empty. We choose $M_{\xi}$ in this set and let $F_{\xi}$ be the translate of $F$ through $M_{\hat{\xi}}$. We claim that the set $D=E-\alpha$ is a $j^{\text {th }}$ minimal hull for $C$ which meets each analytic subset in at most a countable number of points. To show that all $j^{\text {th }}$ projections of $D$ coincide with those of $C$, it is enough to show that the $j^{\text {th }}$ visual hull of $D$ contains $C$. Let $x$ be a point of $C$ and let $P$ be an $(n-j)$-flat through $x$. Then $P$ is amongst $\left\{H_{\xi}\right\}_{\xi<\Omega}$, say $P=H_{\xi^{\prime}}$. If $M_{\xi^{\prime}} \neq \alpha$ then $M_{\xi^{\prime}} \in D \cap H_{\xi^{\prime}}$. If $M_{\xi^{\prime}}=\alpha$ then $\exists M_{\xi^{\prime \prime}}, \xi^{\prime \prime}<\xi^{\prime}$, such that $M_{\xi^{\prime \prime}} \in D \cap H_{\xi^{\prime}}$. In either case $P$ meets $D$ and so $x \in H_{j}(D)$.

If $D$ is not minimal then there exists $M_{\xi}, \xi<\Omega$, such that

$$
H_{j}\left(D-M_{\xi}\right)=C \text {. }
$$

But, projecting $C$ and $D-M_{\xi}$ onto the orthogonal complement of $F$ we see that by construction $\operatorname{proj} C \cap \operatorname{proj} F_{\xi} \neq \varnothing$, but proj $\left(D-M_{\xi}\right) \cap$ $\operatorname{proj} F_{\xi}=\varnothing$. Hence $D$ is a $j^{\text {th }}$ minimal hull for $C$. Finally, suppose that $B$ is an uncountable analytic subset of $D$. If $B$ has positive $j$ dimensional measure then it is possible to find an uncountable analytic subset of $B$ of zero $j$-dimensional measure. Hence it can be supposed that $B$ has zero $j$-dimensional measure and so $B=A_{\xi}$ for some $\xi<\Omega$. But $A_{\xi}=A_{\xi} \cap D \subset \bigcup_{\xi^{\prime}<\xi} M_{\xi^{\prime}}$, which is countable; contradiction.

Of course, if $G$ is an open or compact set in $R^{n}$ then $H_{j}(G)$ will accordingly be an open or compact set. Apart from these cases it does not seem entirely trivial to determine the nature of $H_{j}(G)$ for a given subset $G$ of $R^{n}$. Here we prove the following

Theorem 8. (i) There exists, in the plane $R^{2}$, a borel set $C$ such that $H_{1}(C)$ is analytic but not borel. 


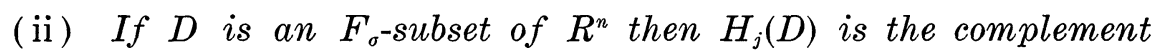
of an analytic set.

REMARKs. We note that by (i) if $C$ is analytic then $H_{1}(C)$ is not necessarily the complement of an analytic set. To disprove the statement that whenever $A$ is analytic then $H_{j}(A)$ is analytic, it would be enough, using (ii), to find an $F_{\sigma}$-subset $D$ of $R^{n}$ such that $H_{j}(D)$ is not borel. (Notice that, a subset, $M$ of $R^{n}$ is borel if and only if $M$ and $R^{n}-M$ are both analytic. Compare, for example, [5]).

Proof. ( i ) As already observed, every analytic set in $R^{1}$ can be represented as the projection into $R^{1}$ of some $F_{\sigma \delta}$ set in $R^{2}$. Let $A$ be an analytic subset of $R^{1}$ such that $A$ is not a borel set and let $B$ be an $F_{\sigma o}$ set in $R^{2}$ such that $\operatorname{proj} B=A$. Take $C$ to be the union of $B$ and the " $y$-axis" $\left(R^{1}\right)^{\perp}$. Then it is easily seen that $H_{1}(C)$ is the union of all lines which are parallel to $\left(R^{1}\right)^{\perp}$ and contain a point of $C$. However this is not a borel set as $H_{1}(C) \cap R^{1}=A \cup\{(0,0)\}$ is not a borel set.

(ii) We define a complete separable metric space $\Omega$, whose points are the $(n-j)$-flats of $R^{n}$, as follows. For each $(n-j)$-flat $F$ in $R^{n}$ let $y$ be the nearest point of $F$ to 0 and set $F \cap\left(S^{n-1}+y\right)=\hat{F}$. Then the distance $\rho\left(F, F^{\prime}\right)$ of two $(n-j)$-flats in $\Omega$ is defined as the Hausdorff distance of $\hat{F}, \hat{F}^{\prime}$ in $R^{n}$. Let $D \subset R^{n}$ be an $F_{\sigma}$ set, say $D=$ $\bigcup_{i=1}^{\infty} D_{i}$ with $D_{i} \subset D_{i+1}$, each $D_{i}$ compact, $i=1,2, \cdots$ Let $D_{i}^{*}, i=$ $1,2 \cdots$ denote the closed subsets of $\Omega$ such that $F \in D_{i}^{*}$ if $F$ meets $D_{i}$ in $R^{n}$. Similarly defined, relative to $D$, is $D^{*}$. Then $D^{*}=\bigcup_{i=1}^{\infty} D_{i}^{*}$ and so $D^{*}$ is an $F_{\sigma}$ subset of $\Omega$. Hence $\Omega-D^{*}$ is a $G_{o}$ set and so, in particular, $\Omega-D^{*}$ is an analytic subset of $\Omega$. Set

$$
\Omega-D^{*}=\sum_{i \in \Lambda} \bigcap_{p=1}^{\infty} A(i \mid p),
$$

where the $A(i \mid p), p=1,2, \cdots$, form a decreasing sequence of compact subsets of $\Omega$, for each $i \in I$. Set

$$
B_{m}=\left\{x \mid x \in R^{n},-m \leqq x_{i} \leqq m, i=1, \cdots, n\right\} .
$$

Let $K_{m}(i \mid p)$ be the closed subset of $B_{m}$ such that $x \in K_{m}(i \mid p)$ if $x$ is contained in an $(n-j)$-flat $F$ with $F \in A(i \mid p)$. Similarly, we define $K_{m} \subset B_{m}$ relative to $\Omega-D^{*}$. Then $K_{m}=\sum_{i \in I} \bigcap_{p=1}^{\infty} K_{m}(i \mid p)$ is an analytic subset of $R^{n}$ and so, therefore, is $K=\bigcup_{m=1}^{\infty} K_{m}$. We claim that $H_{j}(D)=R^{n}-K$. If $x \in K$ then $x \in K_{m}$ for some $m$ and so $x$ is contained in some $\left(n-j\right.$ )-flat $F$ which is contained (in $\Omega$ ) in some set $\bigcap_{p=1}^{\infty} A(i \mid p)$. Hence $F \in \Omega-D^{*}$ which means that $F$ does not meet $D$; i.e., $x \notin H_{j}(D)$. Therefore $R^{n}-K \supset H_{j}(D)$. Conversely if $x \notin H_{j}(D)$ then there exists an $(n-j)$-flat $F$ through $x$ such that $F$ does not meet $D$. Hence $F \in \Omega-$ 
$D^{*}$ and so $F \in \bigcap_{p=1}^{\infty} A(i \mid p)$ for some $i \in I$. Hence $x \in \bigcap_{p=1}^{\infty} K_{m}(i \mid p)$ for some positive integer $m$, i.e., $x \in K$. Therefore $R^{n}-K \subset H_{j}(D)$ and so $H_{j}(D)=R^{n}-K$ is the complement of the analytic set $K$.

Definition. An irregular point $x$ of some closed convex set $C$ in $R^{3}$ is an extreme point $x$ of $C$ such that $x$ lies in two distinct 1-faces $l_{1}, l_{2}$ of $C$, with neither of $l_{1}, l_{2}$ being contained in a 2-face of $C$. Let $C$ be a closed subset of a simple closed curve in the plane $O X Y$. We say that a set $B \subset C \times(-\infty, \infty)$ is vertically convex if every line which is perpendicular to $O X Y$ meets $B$ in a (possibly empty) line segment. We shall make use of the following immediate corollary to a theorem of $\mathrm{K}$. Kunugui [7].

Lemma 3. (Kunugui) Let $B$ be a vertically convex borel set in $C \times(-\infty, \infty)$. Then the projection of $B$ into $C$ is a borel set.

As an immediate consequence of Lemma 3, we have

Lemma 4. Let $B$ be a vertically convex borel subset of some vertically convex closed subset $D$ in $C \times(-\infty, \infty)$. Then the set $D \cap$ $\{($ proj. $B) \times(-\infty, \infty)\}$ is a vertically convex borel set.

In [9] the authors have derived properties of visual hulls for the class of convex sets. Our contribution in this direction is

THEOREM 9. (i) If $C$ is a convex borel (analytic) set in $R^{3}$ then $H_{2}(C)$ is a borel (analytic) set.

(ii) If $C$ is a convex borel (analytic) set in $R^{3}$ and $\bar{C}$ does not have irregular points then $H_{1}(C)$ is a borel (analytic) set.

Proof. (i) We first show that if $C$ is a convex borel (analytic) set in $R^{2}$ then $H_{1}(C)$ is a borel (analytic) set. If $\operatorname{dim} C=1$ then the result is trivial and so it can be supposed that $\operatorname{dim} C=2$. Note that $C^{0} \subset H_{1}(C) \subset \bar{C}$. Let the 1-faces of $\bar{C}$ be $\left\{F_{i}\right\}_{i=1}^{\infty}$. Then

$$
H_{1}(C) \cap\left(\bar{C}-\bigcup_{i=1}^{\infty} F_{i}\right)=C-\bigcup_{i=1}^{\infty} F_{i},
$$

which is a borel set. Let $\left\{F_{i_{2}}\right\}_{\nu=1}^{\infty}$ be the 1-faces of $\bar{C}$ which meet $C$. Then relint $F_{i_{\nu}} \subset H_{1}(C) \cap F_{i_{\nu}}, \nu=1,2, \ldots$. The two endpoints of $F_{i_{\nu}}$ may, or may not, be in $H_{1}(C)$. Nevertheless, $H_{1}(C)$ differs from the borel set $\left(C-\bigcup_{i=1}^{\infty} F_{i}\right) \cup \bigcup_{\nu=1}^{\infty}$ relint $F_{i_{\nu}}$ by at most a countable number of points. And so $H_{1}(C)$ is a borel set. Similarly, if $C$ is a convex analytic set in $R^{2}$, then $H_{1}(C)$ is an analytic set. Suppose now that $C$ is a convex borel set in $R^{3}$. If $\operatorname{dim} C \leqq 2$ then $H_{2}(C)=C$, and so 
it can be supposed that $\operatorname{dim} C=3$. Let $\left\{F_{i}\right\}_{i=1}^{\infty}$ be an enumeration of the 2-faces of $\bar{C}$. Then each $F_{i}$ is closed and $H_{2}(C) \cap\left(\bar{C}-\bigcup_{i=1}^{\infty} F_{i}\right)=$ $C \cap\left(\bar{C}-\bigcup_{i=1}^{\infty} F_{i}\right)$, which is a borel set. As $H_{2}(C) \subset \bar{C}$, it is now enough to show that $H_{2}(C) \cap F_{i}$ is a borel set for $i=1,2, \ldots$. Let $H_{1}^{\prime}\left(C \cap F_{i}\right)$ denote the first visual hull of $C \cap F_{i}$ relative to aff $F_{i}$. Then, from above, $H_{1}^{\prime}\left(C \cap F_{i}\right)$ is a borel set. Let $\left\{F_{i_{j}}\right\}_{j=1}^{\infty}$ be an enumeration of the 1-faces of $F_{i}$. Then $H_{2}(C) \cap\left(F_{i}-\bigcup_{j=1}^{\infty} F_{i_{1}}\right)=H_{1}^{\prime}\left(C \cap F_{i}\right)-\bigcup_{j=1}^{\infty} F_{i_{j}}$ which is a borel set $K_{i}$, say. Let $\left\{F_{i_{j} \nu}\right\}_{\nu=1}^{\infty}$ be the 1-faces of $F_{i}$ which meet $C$ and have the property that the only plane of support to $\bar{C}$ which contains $F_{i_{j \nu}}$ is aff $F_{i}$. Then relint $F_{i_{j \nu}} \subset H_{2}(C)$ and the end points of $F_{i_{j}}$ may or may not be in $H_{2}(C)$. Hence $H_{2}(C) \cap F_{i}$ differs from the borel set $K_{i} \cup\left(\bigcup_{\nu=1}^{\infty}\right.$ relint $\left.F_{i_{j} \nu}\right) \cup\left(\bigcup_{j=1}^{\infty}\left(F_{i_{j}} \cap C\right)\right)$ by at most a countable number of points. Therefore $H_{2}(C) \cap F_{i}$ is a borel set, and so, therefore, is $H_{2}(C)$. Similarly, it can be shown that if $C$ is a convex analytic set in $R^{3}$ then $H_{2}(C)$ is an analytic set.

(ii) Again we shall prove the result for convex borel sets, and indicate at the end the modifications required for convex analytic sets. Let $\left\{r_{i}\right\}_{i=1}^{\infty}$ be an enumeration of the rational numbers and let $P_{i k}$ denote the 2-flat $\left\{x \mid x_{k}=r_{i}\right\} k=1,2,3 ; i=1,2, \ldots$. For each $i, j, k$, let $B(i, j, k)$ denote the closed set formed by the point set union of all maximal line segments in $\bar{C}-C^{0}$ which meet both both $P_{i k}$ and $P_{j k}$. Let $\left\{G_{m}\right\}_{m=1}^{\infty}$ be the 2-faces of $\bar{C}$. If $a 2$-face $G_{m}$ of $\bar{C}$ meets $B(i, j, k)$ then $G_{m}$ meets $C_{i}\left(C_{i}=\left(\bar{C}-C^{0}\right) \cap P_{i k}\right)$ and $C_{j}\left(C_{j}=\left(\bar{C}-C^{0}\right) \cap P_{j k}\right)$ in line segments $1_{i m}$ and $1_{j m}$ respectively. Let $1_{m}^{1}, 1_{m}^{2}$ denote the (at most) two maximal line segments in $G_{m}$ such that each segment contains an endpoint of $1_{i m}$ and $1_{j m}$ but $1_{m}^{1}$ and $1_{m}^{2}$ do not intersect except possibly at end points. Set $C^{*}=\left(\bar{C}-C^{0}\right) \cap P$, where $P$ is a plane parallel to $P_{i k}$ and lying strictly between $P_{i k}$ and $P_{j k}$. Then $G_{m}$ cuts $C^{*}$ in an interval $I_{m}$. Let $1_{m}$ denote the subinterval of $I_{m}$ with endpoints $1_{m}^{1} \cap C^{*}, 1_{m}^{2} \cap C^{*}$, and let $1_{m}^{0}$ be the relative interior of $1_{m}$. Then

$$
C^{\prime}=B(i, j, k) \cap\left(C^{*}-\bigcup_{m=1}^{\infty} 1_{m}^{0}\right)
$$

is a closed subset of $C^{*}$. If $x \in C^{\prime}$, let $\widehat{x}$ denote the unique maximal line segment in $B(i, j, k)$ which passes through $x$ and meets $C_{1}$ and $C_{2}$. Let $X$ denote the closed set formed by the point set union of the line segments $\hat{x}, x \in C^{\prime}$, and set $Q(i, j, k)=\left\{y \mid y \in X, \exists x \in C^{\prime}, \hat{x} \cap C \neq \varnothing, y \in \hat{x}\right\}$. We now show that $Q(i, j, k)$ is a borel set. Every point $y$ of $X$ can be given a coordinate vector $y=\langle x, h\rangle$, where $y \in \hat{x}$ and $h$ is the height, relative to the $j^{\text {th }}$ coordinate, of $y$ above $C^{*}$. Because $\bar{C}$ does not have irregular points, the number of points $y$ in $X$ which receive two different coordinate vectors is countable. Let $\Phi$ be the mapping $X \rightarrow C^{*} \times(-\infty, \infty)$ defined by taking $\Phi\langle x, h\rangle=(x, h), x \in C^{\prime}$. Then $K$ is a borel subset of $X$ if and only if $\Phi(K)$ is a borel subset of the 
closed set $\Phi(X)$. Hence $\Phi(C \cap X)$ is a vertically convex borel subset of $C^{\prime} \times(-\infty, \infty)$. Hence the set $D=X \cap\{\operatorname{proj} \Phi(C \cap X) \times(-\infty, \infty)\}$ is a convex borel set and so $Q(i, j, k)=\Phi^{-1}(D)$ is a borel set. Hence the set $R(i, j, k)=Q(i, j, k)-\bigcup_{m=1}^{\infty} G_{m}$ is a borel set. Consider now the set $S=\bigcup_{i, j, k} R(i, j, k)$ and consider the borel set $T$ defined as the point set union of all 1-faces of $\bar{C}$ which are not contained in some 2-face of $\bar{C}$. We assert that the set $H_{1}^{1}(C)=H_{1}(C) \cap\left(T-\bigcup_{m=1}^{\infty} G_{m}\right)$ equals $S$. For if $y \in H_{1}^{1}(C)$ then, because $\bar{C}$ does not have any irregular points, there exists a unique 1 -face $l$, not contained in $\bigcup_{m=1}^{\infty} G_{m}$, such that $y \in l$. Then $y \in H_{1}(C)$ if and only if $l \cap C=\varnothing$, which happens if and only if $l \subset Q(i, j, k)$ or in other words $y \in R(i, j, k)$ for some $i, j, k$. Hence $H_{1}^{1}(C)=S$. Let $V$ denote the borel set of exposed points of $\bar{C}$ and $H_{1}^{2}(C)=V \cap H_{1}(C), H_{1}^{3}(C)=\bigcup_{m=1}^{\infty}\left(H_{1}(C) \cap\left(G_{m}-V\right)\right)$. Now $H_{1}(C)=H_{1}^{1}(C) \cup H_{1}^{2}(C) \cup H_{1}^{3}(C) . \quad H_{1}^{1}(C)=S$ is a borel set and, since $H_{1}^{2}(C)=V \cap C, H_{1}^{2}(C)$ is a borel set. Hence it is enough to show that $H_{1}(C) \cap\left(G_{m}-V\right)$ is a borel set for all $m$. Now let $\left\{G_{m_{\nu}}\right\}_{\nu=1}^{\infty}$ be those 2 -faces of $\bar{C}$ which meet $C$. Then relint $G_{m_{\nu}} \subset H_{1}^{3}(C)$ for all $\nu$. Let $\left\{G_{m_{\nu} n}\right\}_{n=1}^{\infty}$ be the 1-faces of $G_{m_{\nu}}$. Then either relint $G_{m_{\nu} n} \subset H_{1}^{3}(C)$ or relint $G_{m_{\nu} n} \cap H_{1}^{3}(C)=\varnothing$. Then the endpoints of $G_{m_{\nu} n}$ may or may not be in $H_{1}^{3}(C)$. Let $H_{m}$, be the countable set of those endpoints of $\left\{G_{m_{\nu} n}\right\}_{\nu=1}^{\infty}$ which lie in $H_{1}^{3}(C)$ and let $\left\{G_{m_{\nu} n_{\mu}}\right\}_{\}^{\prime}=1}^{\infty}$ be the 1-faces of $G_{m_{\nu}}$ whose relative interiors are contained in $H_{1}^{3}(C)$. We have $G_{m_{\nu}} \cap H_{1}^{3}(C)=$ relint $G_{m_{2}} \cup\left(\bigcup_{\mu=1}^{\infty}\right.$ relint $\left.G_{m_{2} n_{\mu}}\right) \cup H_{m_{2}}$, which is a borel set. If, on the other hand, a 2 -face of $\bar{C}$ does not meet $C$, its intersection with $H_{1}^{3}(C)$ is empty. Therefore $H_{1}^{3}(C) \cap G_{m}$ is a borel set for all $m$, and $H_{1}(C)$. is a borel set.

For the case when $C$ is an analytic set, say $C=\sum_{i \in I} \bigcap_{n=1}^{\infty} C(i \mid n)$ in the usual representation, the only modification required to the above proof is to show that the set $Q(i, j, k)$ is an analytic set. With the previous notation, $Q(i \mid n)=\left\{y \mid y \in X, \exists x \in C^{\prime}, \hat{x} \cap C(i \mid n) \neq \varnothing, y \in \hat{x}\right\}$. Then $Q(i \mid n)$ is a closed set and $Q(i, j, k)=\sum_{i \in I} \bigcap_{n=1}^{\infty} Q(i \mid n)$. Therefore $Q(i, j, k)$ is an analytic set.

\section{REFERENCES}

1. W. A. Beyer, The visual hull of a polyhedron, Proceedings of the Conference on Projections and related Topics, Clemson University, Clemson, South Carolina, 1968.

2. W. A. Beyer and S. Ulam, Note on the visual hull of a set, J. of Comb. Theory 2 (1967), 240-245.

3. N. Bourbaki, Eléments de mathématique, livre VI, Paris, 1963.

4. B. Grünbaum, Convex Polytopes, Wiley, 1967.

5. W. Hurewicz, Zur Theorie der analytischen Mengen, Fund. Math. 15 (1930), 8.

6. V. L. Klee, Some characterizations of convex polyhedra, Acta Math. 102 (1959), 79-107.

7. K. Kunugui, Sur un problème de M. E. Szpilrajn, Proc. Imp. Acad. Tokyo, 16 (1940). 73-78. 
8. C. Kuratowski, Topologie I, $4^{\text {th }}$ ed., Warszawa 1958.

9. G. H. Meisters and S. Ulam, On visual hulls of sets, Proc. Nat. Acad. Sci. 57 (1967), 1172-1174.

Received March 12, 1969, and in revised form May 15, 1969. The first author was supported by a Harkness Fellowship of the Commonwealth Fund and the second author by a Fellowship from Swiss National Foundation.

University College, London, England

AND

Universitaet Bern, Switzerland 



\section{PACIFIC JOURNAL OF MATHEMATICS}

\section{EDITORS}

\author{
H. SAMELSON \\ Stanford University \\ Stanford, California 94305 \\ Richard Pierce \\ University of Washington \\ Seattle, Washington 98105
}

J. DUGUNDJI

Department of Mathematics

University of Southern California

Los Angeles, California 90007

BASIL GORDON*

University of California

Los Angeles, California 90024

\section{ASSOCIATE EDITORS}
E. F. BECKENBACH
B. H. NeUmanN
F. WOLF
K. YoSHIDA

\section{SUPPORTING INSTITUTIONS}

UNIVERSITY OF BRITISH COLUMBIA CALIFORNIA INSTITUTE OF TECHNOLOGY

UNIVERSITY OF CALIFORNIA MONTANA STATE UNIVERSITY

UNIVERSITY OF NEVADA

NEW MEXICO STATE UNIVERSITY

OREGON STATE UNIVERSITY

UNIVERSITY OF OREGON

OSAKA UNIVERSITY

UNIVERSITY OF SOUTHERN CALIFORNIA
STANFORD UNIVERSITY

UNIVERSITY OF TOKYO

UNIVERSITY OF UTAH

WASHINGTON STATE UNIVERSITY

UNIVERSITY OF WASHINGTON

AMERICAN MATHEMATICAL SOCIETY CHEVRON RESEARCH CORPORATION TRW SYSTEMS

NAVAL WEAPONS CENTER 


\section{Pacific Journal of Mathematics}

\section{Vol. 32, No. $1 \quad$ January, 1970}

Robert Alexander Adams, Compact Sobolev imbeddings for unbounded domains ........................................ 1

Bernhard Amberg, Groups with maximum conditions .................. 9

Tom M. (Mike) Apostol, Möbius functions of order k............... 21

Stefan Bergman, On an initial value problem in the theory of two-dimensional transonic flow patterns ................... 29

Geoffrey David Downs Creede, Concerning semi-stratifiable spaces ...... 47

Edmond Dale Dixon, Matric polynomials which are higher

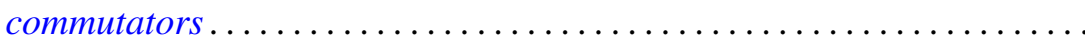

R. L. Duncan, Some continuity properties of the Schnirelmann density.

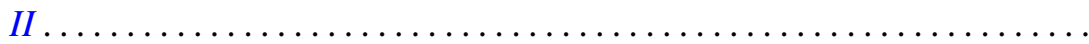

Peter Larkin Duren and Allen Lowell Shields, Coefficient multipliers of $H^{p}$

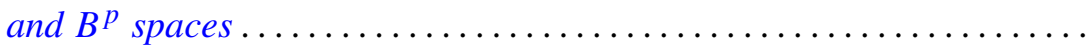

Hector O. Fattorini, On a class of differential equations for vector-valued

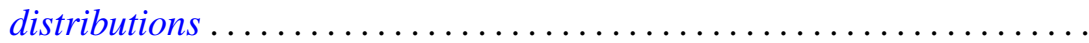

Charles Hallahan, Stability theorems for Lie algebras of derivations. . . . . . 105

Heinz Helfenstein, Local isometries of flat tori ................ 113

Gerald J. Janusz, Some remarks on Clifford's theorem and the Schur

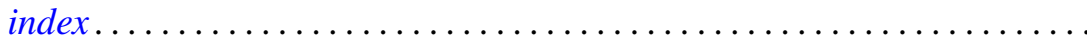

Joe W. Jenkins, Symmetry and nonsymmetry in the group algebras of discrete groups. ...

Herbert Frederick Kreimer, Jr., Outer Galois theory for separable

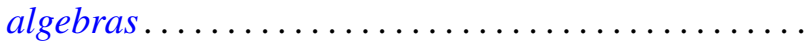

D. G. Larman and P. Mani, On visual hulls

R. Robert Laxton, On groups of linear recurrences. II. Elements of finite order.

Dong Hoon Lee, The adjoint group of Lie groups ...

James B. Lucke, Commutativity in locally compact rings

Charles Harris Scanlon, Rings of functions with certain Lipschitz

$$
\text { properties ............................... }
$$

Binyamin Schwarz, Totally positive differential systems .

James McLean Sloss, The bending of space curves into piecewise helical curves.

James D. Stafney, Analytic interpolation of certain multiplier spaces ...

Patrick Noble Stewart, Semi-simple radical classes.......

Hiroyuki Tachikawa, On left $\mathrm{QF}-3$ rings ...................... 255

Glenn Francis Webb, Product integral representation of time dependent nonlinear evolution equations in Banach spaces.... . . . 